\title{
BU LLETIN
}

OF

\section{ENTOMOLOGICAL RESEARCH}

ISSUED BY THE IMPERLAL
BUREAU OF ENTOMOLOGY.

EDITOR: THE DIRECTOR.

VOL. VII.

LO N D O N :

SOLD BY

DULAU \& Co., LTD., 37, SOHO SQUARE, W. 1. 1916-1917. 


\title{
IMPERIAL BUREAU OF ENTOMOLOGY. Gonorary Committee of management.
}

\author{
VISCOUNT HARCOURT, M.P., Chairman.
}

Lieutenant-Colonel A. W. ALCock, C.I.E., F.R.S., London School of Tropical Medicine.

Mr. E. E. AUSTeN, Entomological Department, British Museum (Natural History).

Dr. A. G. Bagshawe, C.M.G., Director, Tropical Diseases Bureau.

Mr. E. C. BLECH, C.M.G., Foreign Office.

Sir J. Rose Bradford, K.C.M.G., F.R.S., Secretary, Royal Society. Surgeon-General Sir David Bruce, C.B., F.R.S., A.M.S.

Mr. J. C. F. Fryer, Entomologist to the Board of Agriculture and Fisheries.

Dr. S. F. Harmer, F.R.S., Keeper of Zoology, British Museum (Natural History).

Professor H. MAXwell LeFroy, Imperial College of Science and Technology.

The Hon. Sir JoHn McCaLl, M.D., Agent-General for Tasmania.

Dr. R. Stewart MacDougall, Lecturer on Agricultural Entomology, Edinburgh University.

Sir John McFadyean, Principal, Royal Veterinary College, Camden Town.

Sir Patrick Manson, G.C.M.G., F.R.S., Late Medical Adviser to the Colonial Office.

Sir Daniel Morris, K.C.M.G., Late Adviser to the Colonial Office in Tropical Agriculture.

Professor R. Newstead, F.R.S., Dutton Memorial Professor of Medical Entomology, Liverpool University.

Professor G. H. F. NutTale, F.R.S., Quick Professor of Protozoology, Cambridge.

Professor E. B. Poulton, F.R.S., Hope Professor of Zoology, Oxford.

Lieutenant-Colonel Sir David Prain, C.I.E., C.M.G., F.R.S., Director, Royal Botanic Gardens, Kew.

Mr. H. J. Read, C.B., C.M.G., Colonial Office.

The Honourable N. C. Rothschild.

Mr. Hugh ScotT, Curator in Entomology, Museum of Zoology, Cambridge.

Dr. A. E. Shipley, F.R.S., Master of Christ's College, Cambridge.

Sir Stewart Stockman, Chief Veterinary Officer, Board of Agriculture.

Mr. F. V. Theoвald, Vice-Principal, South-Eastern Agrioultural College, Wye.

Mr. C. Warburton, Zoologist to the Royal Agricultural Society of England.

The Chief Entomologist in each of the Self-Governing Dominions is an ex officio member of the Committee.

General secretary.

Mr. A. C. C. Parkinson (Colonial Office).

Director ano Eoitor.

Dr. Guy A. K. Marshall.

Zissistant Đirector.

Mr. S. A. NeAve.

Ilead Office.-British Museum (Natural History), Cromwell Road, London, S.W. 7.

Publication Office.-S9, Queen's Gate, Iondon, S.W. T 


\section{CONTENTS.}

\section{ORIGINAL ARTICLES.}

ADERS, DR. W. M. PAGE.

Insects injurious to man and stock in Zanzibar............ 391

Braner, Prof. M.

On the fruit-flies of the genus Dacus (s.l.) occurring in India, Burma, and Ceylon.............................. 99

Bodkin, G. E., and Cleare, L. D., Jnr.

Notes on some animal parasites in British Guiana........... 179

Cameron, Dr. Alfred E.

Some experiments on the breeding of the mangold fly (Pegomyia hyoscyami, Panz.) and the dock fly (P. bicolor, Wied.)....... 87

Distant, W. L.

On some Rhynchota of economic importance from Colombia..... 381

EDwards, F. W.

Ten new African Haematopota..................... 145

Notes on Culicidae, with descriptions of new species......... 201

Fiske, W. F.

Insects injurious to vegetation. ................... 383

Green, E. Ernest.

Observations on some recently described Coccidae.......... 51

Remarks on Coccidae from Northern Australia............. 53

Notes on Coccidae occurring in the Seychelles Islands, with descriptions of new species $\ldots \ldots \ldots \ldots \ldots \ldots \ldots \ldots \ldots \ldots \ldots \ldots$

Jackson, Miss A. C., and Lefroy, Prof. H. M.

Some fy poisons for outdoor and hospital use............ 327

iC403) Wt P.7/121. 1,000. 11.17. B.\&F.Ltd Gp.11/1. 
Johnston, DR. J. E. L.

PAGE.

A summary of an entomological survey of Kaduna District, Northern

Nigeria

Lamborn, Dr. W. A.

Third report on Glossina investigations in Nyasaland.

LLOYd, Lu.

Report on the investigation into the bionomics of Glossina morsitans

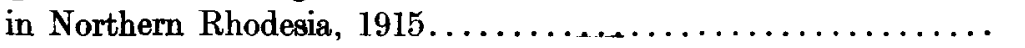

Macdonald, Dr. Angus.

Notes on blood-sucking flies in Grenada............... 259

Macfie, Dr. J. W. ScotT.

The limitations of kerosene as a larvicide, with some observations on the cutaneous respiration of mosquito larvae..........277

Morphological changes observed during the development of the larva of Stegomyia fasciata...................... 297

Macfie, Dr. J. W. Scott, and Ingram, Dr. A.

New Culicine larvae from the Gold Coast............... 1

The domestic mosquitos of Accra .................. 161

MagGregor, Malcolm Evan.

Resistance of the eggs of Stegomyia fasciata (Aëdes calopus) to conditions adverse to development. .................. 81

Marshall, Dr. Guy A. K.

A new weevil attacking pine-apples in Jamaica........... 197

Mautik, S.

Solubility of the scale of Lepidosaphes $u$ umi, Linn . . . . . . . . 267

Newstead, Prof. R.

On the genus Phlebotomius. -Part III. ............... 191

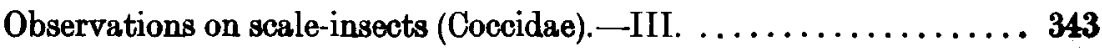

Stanton, A. T., and Hacker, H. P.

The Anopheles of Malaya.-IHI. A new variety of $A$. albotaeniatus, Theo....................................273

Taylor, Frank H.

Sarcophaga frogatti, sp. n. $\rightarrow$ new sheep-maggot fly $\ldots \ldots \ldots \ldots 265$ 
Theobald, Fred V.

The Aphid of tea, coffee and cacao (Toxoptera coffeae, Nietner) .... 337

Turner, R. E.

On Mutillidae parasitic on Glossina morsitans............ 93

Torner, R. E., and Waterston, James.

A new parasite bred from Glossina morsitans in Nyasaland....... 133

Waterston, James.

Notes on African Chalcidoidea. $-\mathrm{V} . \ldots \ldots \ldots \ldots \ldots \ldots \ldots \ldots \ldots$

Notes on Coccid-infesting Chalcidoidea.-I, II, III. .....137, 231, 311

Wirliams, C. B.

Notes on a froghopper attacking sugar-cane at Marienburg Estate, Surinam

\section{MISCELLANEOUS.}

Collections received $\ldots \ldots \ldots \ldots \ldots \ldots \ldots \ldots \ldots \ldots \ldots \ldots . .197,309,403^{\circ}$ 


\section{PLATES.}

PAGE,

1. Views in N. Rhodesia, haunts of Glossina morsitans ... facing 80

II. Wings of African species of Haematopota ......., , 160

III. \& IV. Views in Grenada of haunts of blood-sucking flies ... , , 264

V. Injurious Rhynchota from Colombia ............, , 382

VI. The male of Lecanium hesperidum (Linn.) ...., ., , 380

VII. Pimento trees attacked by scale-insects in Jamaica .. " 380

\section{A'PS.}

I. Map of Accra showing the distribution of mosquitos (1912-1915)

PAGE.

in the various blocks into which the town is divided...... to face 178

II. Map of Accra showing the samples of mosquito larvae collected in native compounds during the year 1915, distributed according to the various blocks into which the town is divided $\ldots \ldots \ldots \ldots \ldots \ldots \ldots \ldots \ldots \ldots \ldots \ldots \ldots \ldots, n \ldots \ldots \ldots \ldots \ldots \ldots, 178$

III. Map of British Guiana revised to $1909 . \ldots \ldots \ldots \ldots \ldots \ldots \ldots, \quad 190$ 


\section{ILLUSTRATIONS IN THE TEXT.}

Hollow tree in which were larvae of Stegomyia metallica, S. unilineata and

Ouliciomyia nebulosa

Details of mosquito larvae :-

Culex ingrami, Edw.

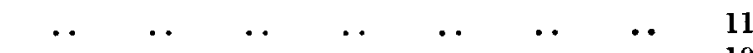

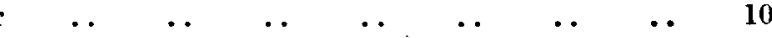

Eumelona, Theo. $\begin{array}{llllllll} & \cdots & \ldots & \ldots & \ldots & \ldots & \ldots & \mathbf{8 , 9}\end{array}$

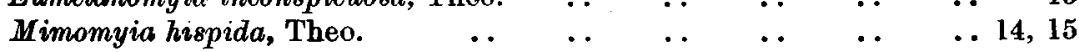

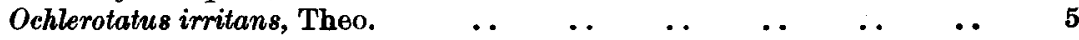

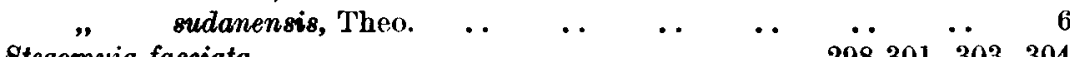

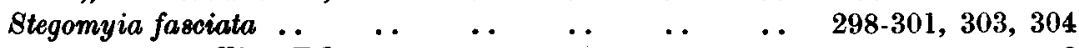

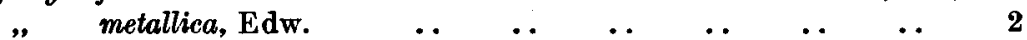

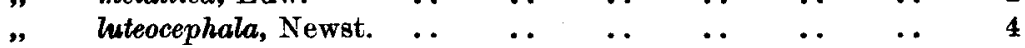

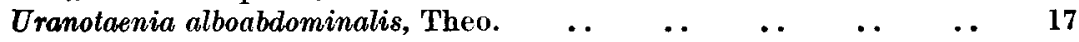

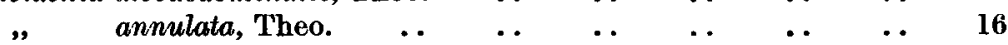

Bketch-msp of the country round Kaduna Bridge, Nigeria $\quad \ldots \quad \ldots \quad \ldots 20,25$

Detaile of scale-insects :-

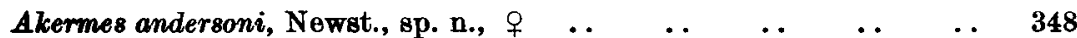

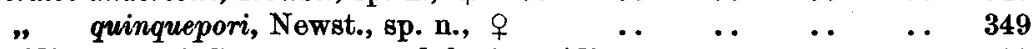

A ppidiotus ansei, Green, sp. n., adult + pygidium $\quad \ldots \quad$. $\quad \ldots \quad \ldots \quad 193$

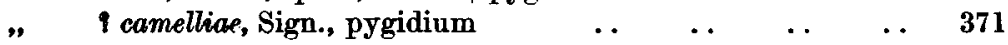

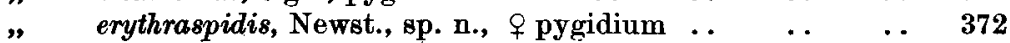

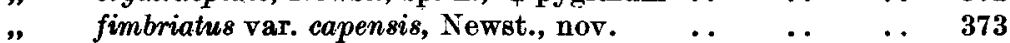

" mauritianus, Newst., sp. n., $\circ$, larva and puparium $\quad . \quad 374$

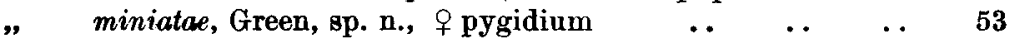

" pimentae, Newst., sp. n., + and larval antenna $\quad \ldots \quad$. $\quad 376$

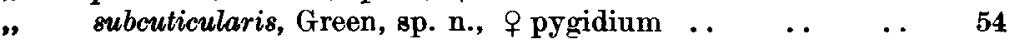

Asterolecanium hilli, Green, sp. n., \& $\quad \ldots \quad$.

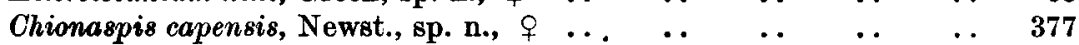

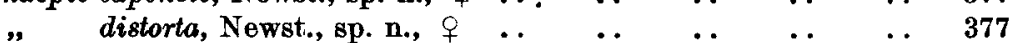

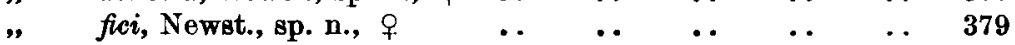

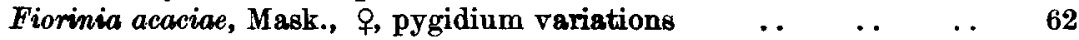

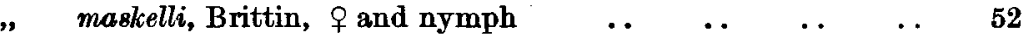

Gymmaspis grandis, Green, sp. n., $\&$, puparia, nymph and larva $\quad \ldots \quad 194$

Hemichionaspis pseudaspidistrae, Green, f pygidium $\ldots \quad \ldots \quad \ldots \quad \ldots \quad 58$

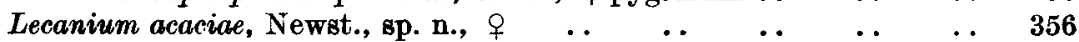

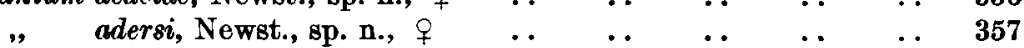

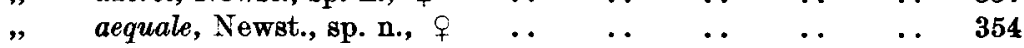

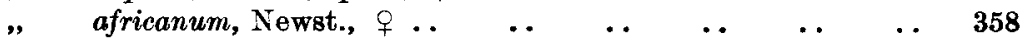

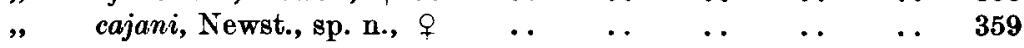


Details of scale-insects :-

Lecanium chelonioides, Newst., sp. n., + , dorsal aspects

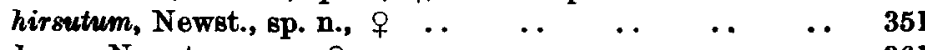

" hurae. Newst., sp. n., o $\quad \ldots \quad$.

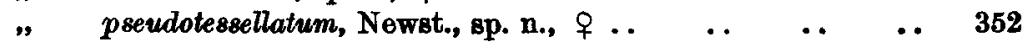

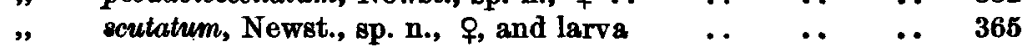

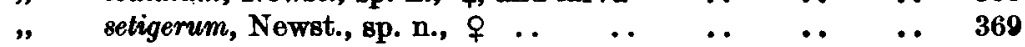

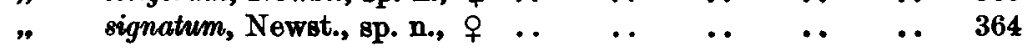

" subhemisphaericum, Newst., sp. n., + antenna $\quad \ldots \quad \ldots \quad 363$

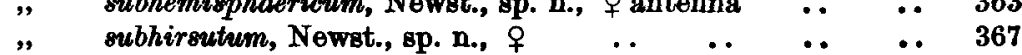

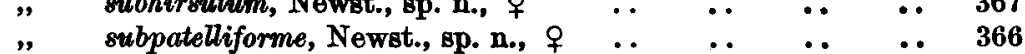

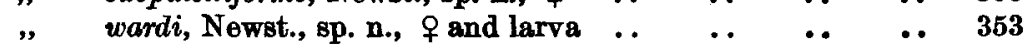

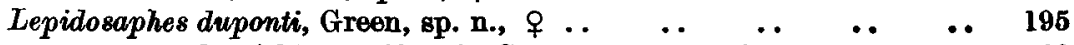

" hemichionaspiformis, Green, sp. n., $\delta$ \% $\quad \ldots \quad$.. 60

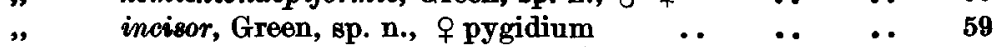

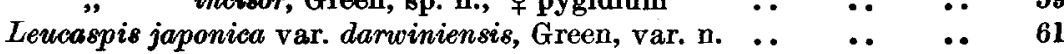

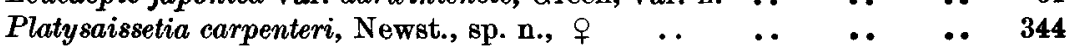

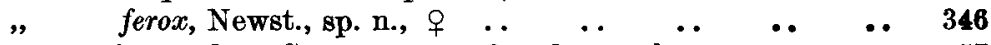

Porogymnaspis angulata, Green, sp. n., $\circ$ and nymph $\quad . . \quad \quad \ldots \quad 57$

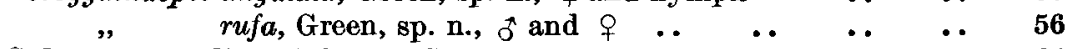

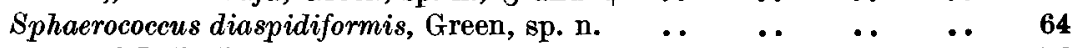

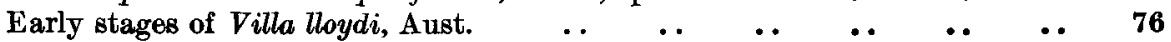

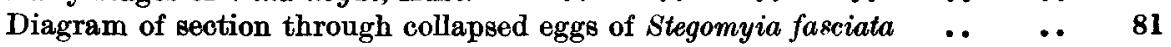

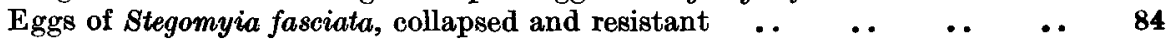

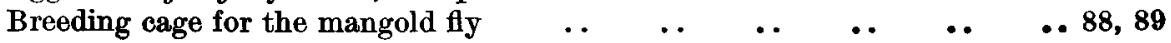

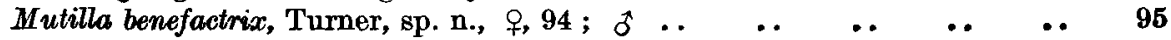

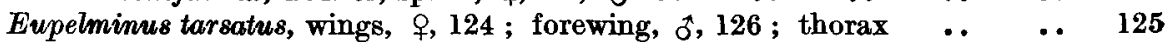

Sycophaga cyclostigma, Waterston, sp. n., o, hind femur and tibia .. $\quad . . \quad 128$

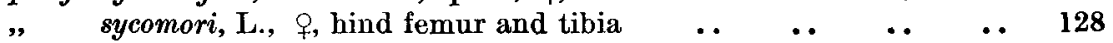

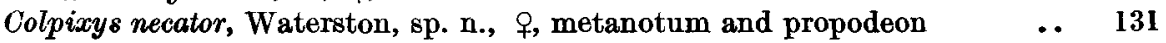

Prolaelius glossinae, Turner and Waterston, sp. n., +, thorax and head, 133 ;

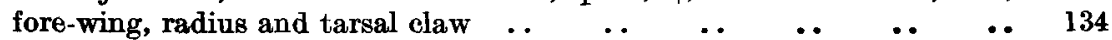

Diversinervus silvestrii, Waterston, sp. n., q, thorax and propodeon, 139;

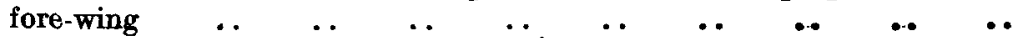

Coccophagus acanthosceles, Waterston, $\ddot{\text { sp. }} \dot{\mathrm{n}}$., thorax and abdomen, mandible,

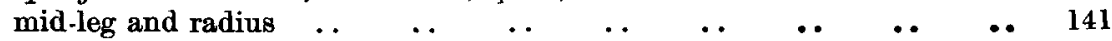

Heads of :-

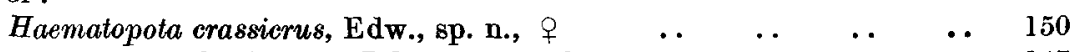

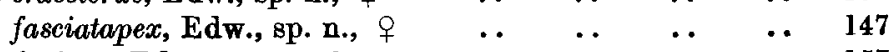

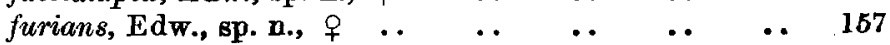

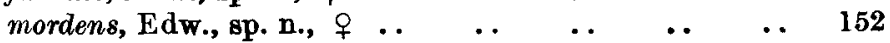

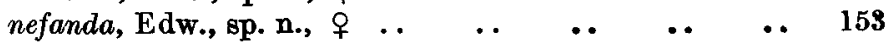

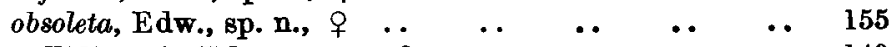

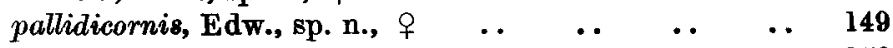

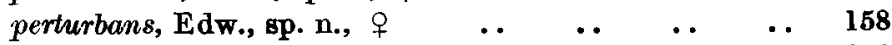

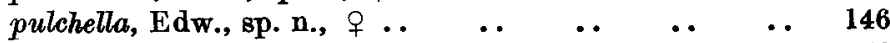

, $\quad$ rabida, Edw., sp. n., $\circ \quad \ldots \quad$.

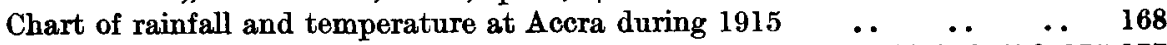

Distribution tables of mosquitos of Accra $\quad . . \quad \ldots \quad 163-166,169,172,175.177$

Tabanus desertus, Walk., + , pupal aster, 184 ; imago $\quad \ldots \quad \ldots \quad \ldots \quad 187$

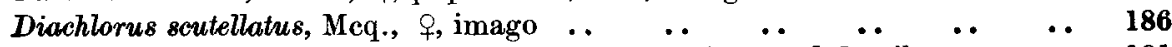

Phlebotomus major var. chinensis, Newst., nov., wings and detailf $\ldots$.. $\quad \ldots \quad 191$

Metamasius ritchiei, Mshl., sp. n., of, dorsal view and details $\quad \ldots \quad \ldots \quad 197$ 
Male genitalia of :-

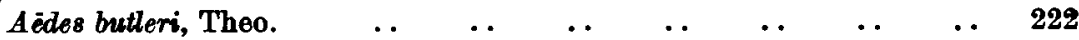

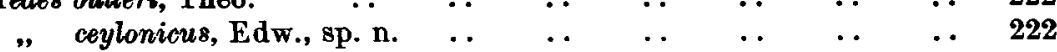

" fragilis, Leic.

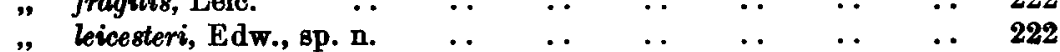

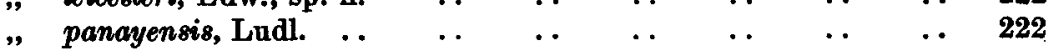

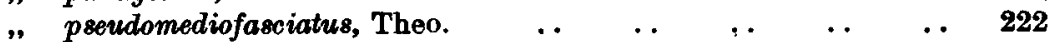

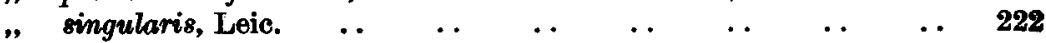

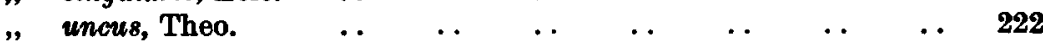

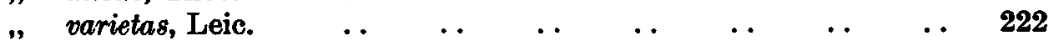

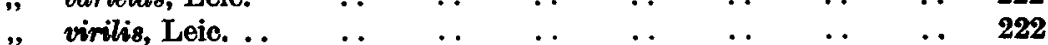

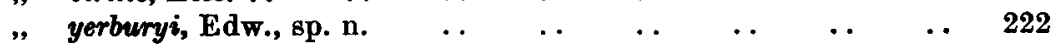

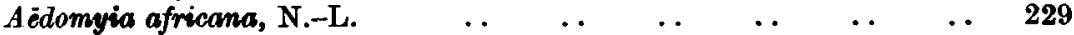

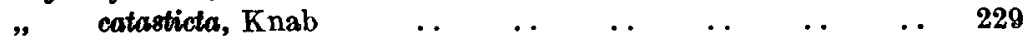

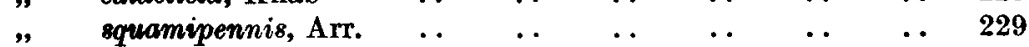

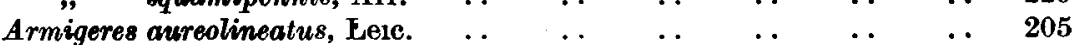

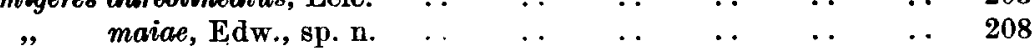

" $\begin{array}{lllllllll}\text { malayi, Theo. } & . & \ldots & \ldots & \ldots & \ldots & \ldots & \ldots & 207\end{array}$

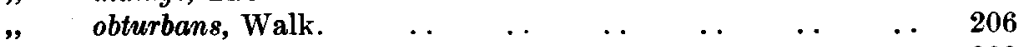

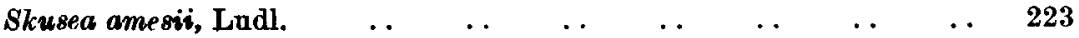

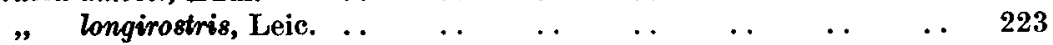

" simplex, Theo. $\quad . \quad$.

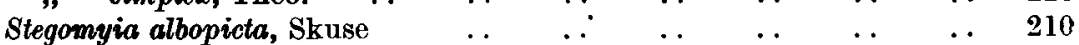

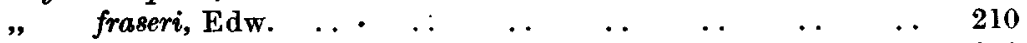

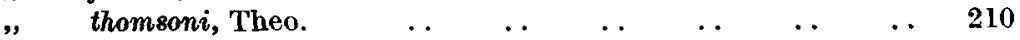

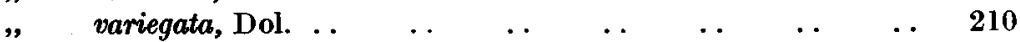

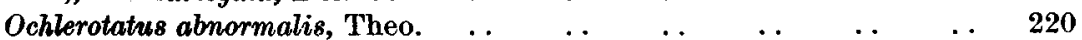

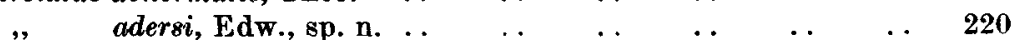

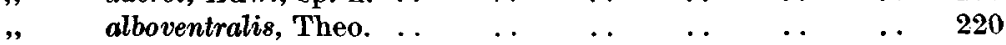

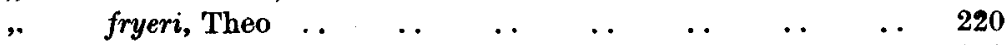

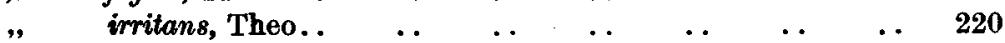

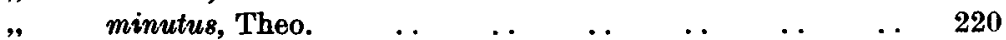

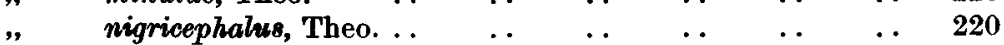

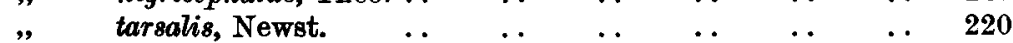

Seales from comb of the eighth abdominal segment of larvae of Ochlerotatus dorsalis, Mg.; $O$. nemorosus, $\mathrm{Mg}$. ; and $O$. salinus, Fic. .. $\quad . . \quad \ldots 216$

Wings, antennae and mandibles of :-

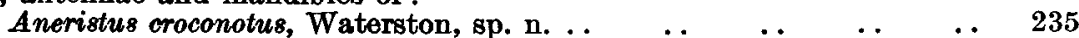

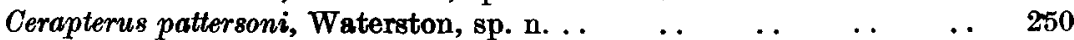

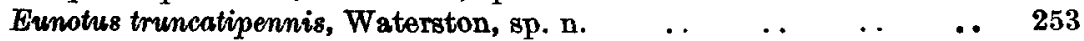

Eusemion cornigerum, Wlk., $\&$.

Coccidoxemus coelops, Waterston, sp. n., + , head and mandible, 239 ; wing.. 241

" obscuratus, Waterston, sp. n., \&, head and mandible, 239; radius of right fore wing $\quad \ldots \quad$.

Ohiloneurus afer, Waterston, sp. n., + , head, 244 ; antenna, 245 ; right fore

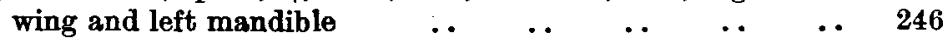

" cyanonotus, Waterston, sp. n., ㅇ, hesd, 244 ; antenna, 245 ; radius of right fore wing $\quad \ldots \quad$.

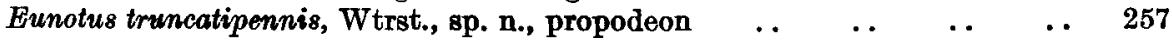

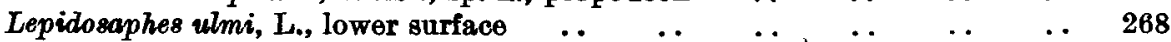

Hind legs of Anopheles albotaeniatus, Theo., and A. albotseniatus var. mon-

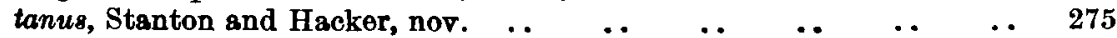

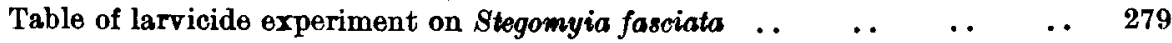


Diagram of apparatus used in larvicide experiment $\quad \ldots \quad \ldots \quad \ldots \quad \ldots \quad \ldots$

Larva of Stegomyia fasciata, head, in four stages, 298 ; labial plate in four stages, 299 ; four stages of ventral thoracic hook and second abdominal segment, 300 ; four stages of scales from comb and from pecten, 301 ; posterior end in first three stages, 303 ; end in fourth stage .. ..

Coccidoxenus distinguendus, Waterston, sp. n., mandible and details of neuration

Aëthognathus afer, Silv., var. cavilabris nov., o labrum $\quad \cdots \quad \ldots$ Eusemion italicum, Masi, 우, basal half of fore wing _. $\quad \ldots \quad \ldots \quad \ldots \quad \ldots \quad 316$

Habrolepis apicalis, Waterston, sp. n., o, antenna, mandibles, and hind femur 319

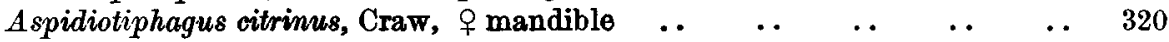
Eriaporus laticeps, Waterston, sp. n., + , mandible, right fore wing and

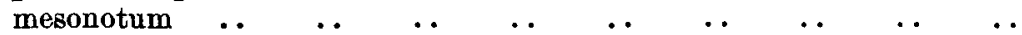

Toxoptera coffeae, Nietn., details of , alate and apterous, 329 ; wings, 340 ;

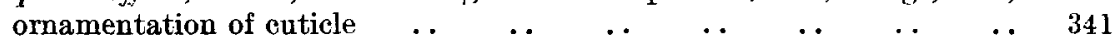

$\begin{array}{llllll}\text { Diagrams of relations between plants and insects } & \ldots & \ldots & \ldots & \ldots & 388\end{array}$

Chart showing seasonal incidence of Tabanidae in Zanzibar $\quad \ldots \quad \ldots \quad \ldots$ 


\section{ER R A T A.}

\begin{tabular}{|c|c|c|c|c|c|}
\hline Page & 51 , & de & 11 & for & p. 74 \\
\hline " & 88, & " & 25 , & פ & "Empis tesselato" \\
\hline ", & 97 & " & 24, & ," & "Mr. M. H. Dawe" \\
\hline , & 120 , & " & 29 , & , & "Prof. Buguion" \\
\hline , & 158 & ", & 23 , & $"$ & "Dr. J. O. Murphy" \\
\hline " & 171, & ", & 22 , & , & "O. nigrocephalus " \\
\hline , & 182, & ", & 36 & ," & "Dicorcelium" \\
\hline , & 183, & ", & 2 & ", & "Echinorynchus" \\
\hline ," & 189, & ", & 35 & , & "donestica" \\
\hline " & 189 & ", & 39 & , & "Crux", \\
\hline ," & 234 & ,", & 13 , & , & "tosPentelicus" \\
\hline & 238 & ", & 29 & ", & "Tephrosia vogilii" \\
\hline & $3 \overline{5}$ & , & 98 & "' & "Coupida" \\
\hline & 363 & "' & & , & "syluestrii" \\
\hline , & $\begin{array}{l}366, \\
400,\end{array}$ & ," & $\begin{array}{l}17, \\
16,\end{array}$ & " & $\begin{array}{l}\text { "Mimusops globosa" } \\
\text { "Xenopsylla cheopsi" }\end{array}$ \\
\hline
\end{tabular}

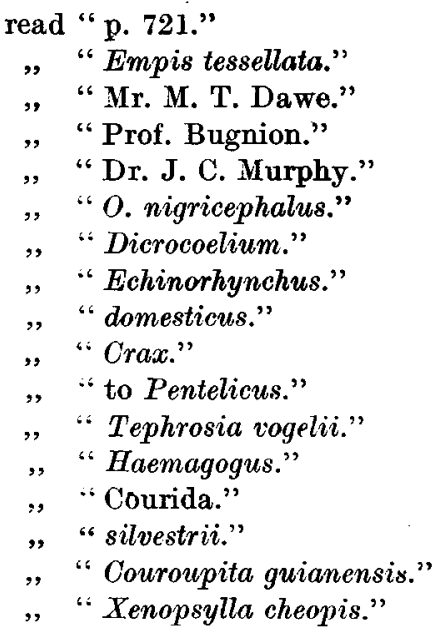

